\title{
User-Centred Design with a Remote Approach: Experiences from the Chronic Pain Project
}

\author{
Berglind F. SMARADOTTIR ${ }^{\mathrm{a}, \mathrm{b}, 1}$, Johan Gustav BELLIKA ${ }^{\mathrm{a}}$, \\ Aina FREDENG ${ }^{\mathrm{a}}$ and Asbjørn J. FAGERLUND ${ }^{\mathrm{a}}$ \\ ${ }^{a}$ Norwegian Centre for E-health Research, University Hospital of North Norway, \\ Tromsø, Norway \\ ${ }^{\mathrm{b}}$ Department of Information and Communication Technology, University of Agder, \\ Grimstad, Norway
}

\begin{abstract}
User-centred design involves end-users or user groups during all the parts of the development process. The research project Chronic Pain aims to develop a shared decision making application for patients and physicians, addressing individually adapted pain treatment. The project employs a user-centred design process, and in middle of it, Covid-19 pandemic social distancing restrictions were imposed. This paper presents how the user-centred design process together with a patient organisation was transformed to a digital approach and the experiences from performing a remote co-creation user workshop. The digital approximation had a satisfactory result and the main contribution lies in the sharing of recommendations for how to practically apply a remote user-centred design methodology.
\end{abstract}

Keywords. User-centred design, video conference, chronic pain management, decision support, digital services

\section{Introduction}

A user-centred design process means involvement of groups of users throughout the entire development cycle [1]. The tasks of the users are to contribute with descriptions of the context of use, elicitation of user needs and being test participants in user tests [2]. These are all contributions for designing and building health information technology through iterations. Workshops are a common way for the collection of user needs and context of use and where potential end-users, often recruited from patient organisations, are gathered together for a half or whole day [3]. A first workshop aims to familiarize with the goal of the development, the other participants, the development- and research team and the commitment of the participation in the user-centred design process. Further, in such a workshop the users are asked to, based on their own experiences, describe context of use, how the use of the technology could support their daily life and in what way to interact with it. The following workshops work as feedback sessions for conceptual design, wireframes or prototypes of technology. During iterative

\footnotetext{
${ }^{1}$ Corresponding author, Department of Information and Communication Technology, Faculty of Engineering and Science, University of Agder, Jon Lilletuns vei 9, N-4879 Grimstad, Norway, E-mail: berglind.smaradottir@uia.no.
} 
development, individual user tests or paired testing are made to frequently evaluate the technology [4].

In the research project Chronic Pain-Decision support for personalised chronic pain care (2019-2021) user-centred design has been applied from the early project idea and is planned for the entire development process of a shared decision making application [5]. The application will provide patients and physicians with relevant and valid decision alternatives, also presenting realistic probabilities for outcomes, side effects and adverse events. Another requirement for the project is to address how to collect and share patientreported outcomes (PROMs) and experiences using a mobile application that utilises distributed data storage. The user-centred design procedure is made in collaboration with the Norwegian Fibromyalgia Association [6], and one user meeting and two user cocreation workshops to elicit context of use and user needs have previously been organised [7]. Four workshops are planned organised for each year, approximately every third month. However, in the middle of the user-centred design process, the Covid-19 pandemic escalated during the spring of 2020 and social distancing restrictions were imposed. All physical meetings were discouraged and cancelled, and the research team involved was obliged to home officing. This would imply a delay for the project, with negative effects on the progress and goals. However, the end-users from the patient organisation was an engaged, enthusiastic and active group, and the research team decided to continue the user-centred design process remotely. This paper reports from how the user-centred design process was transformed from physical to digital meetings, and shares the experiences from a remote co-creation user workshop. The research questions (RQs) stated were:

RQ1: How can a user-centred design process be performed with a remote digital approach?

RQ2: What are the benefits and constraints of performing a remote digital approach in a user-centred design process?

\section{Methodology}

Qualitative methods were used in the Chronic Pain project to analyse the user-centred design process. The project has five steps: 1) user-centred design of the PROMs data collection tools, 2) design data storage and computation environment, 3) technical development of PROMs application, 4) building data storage and computation environment and 5) user evaluations. The project is in the middle of the first step, and earlier two in-house user workshops have been organised, with a duration of 4 hours including a lunch break. A third workshop was planned in June 2020. Due to the Covid19 pandemic restrictions, this workshop was converted to a digital event to remotely gather the project participants. Six participants from the Norwegian Fibromyalgia Association that had attended the previous physical workshops were invited and all accepted to join for the digital event. The workshop was hosted on the browser-based video conferencing platform Whereby (Video Communication Services AS, Maaloy, Norway). The workshop was scheduled with a duration of 2 hours, including a short break. The workshop addressed the topics: storage of research data, demonstration of a web-application and mobile system for pain registration and a session for user feedback. The feedback session targeted the first time user experience regarding the log-in procedure and the interactions for registering pain using a numeric rating scale (NRS). 
The six participants were all female pain patients, with an average age of 54 years and the average of 27.8 years since onset of pain and 15.1 years since diagnosed with fibromyalgia. On a scale from $0-10$, they self-evaluated their technology skills at 6.0 and interest of technology at 6.7. The research team consisted of people with expertise in human factors in design, psychology, statistics, health science and medical informatics. After the remote workshop, there was a 30 minutes long debriefing session for the research team to summarise the outcome and the experiences.

The data collection consisted of audio recordings from the workshop and annotations from the workshop and the debriefing session. The recording was made with an Olympus VN-3200PC audio recorder, physically located at the workshop host. Screen recording was not made due to privacy regulations for data storage. The Privacy Officer at the University Hospital of North Norway approved the study with project number 02147. The participation in the study was voluntary and all informants signed a consent form at the project start. Additionally, a specific consent for audio recording was obtained prior to the remote workshop.

\section{Results}

The results target the 1) technical and practical issues and 2) experiences of the research team from performing the remote user workshop. The overall experience was that the execution of the digital workshop worked in a satisfactory way. There was an active dialogue throughout the entire session. It might have had a positive impact that the participants had met before and were familiar with each other, the project and the research team.

A few days before the workshop user training on Whereby was conducted with each participant individually, testing how to connect, the sound quality, the mute function and the use of the video-camera, to avoid delaying technical issues at the workshop day. Only minor technical issues were experienced during the digital workshop, mainly regarding the sound quality. A useful feature of the videoconference solution was the simultaneous visualisation of all the participants in the screen view (up to 12 persons) and that provided a good overview for the dialogue and discussion between the participants. Even in presentation mode or the share screen function, the participants were shown with a small picture. Compared to other videoconference solutions that allow only a few participants' video to be displayed simultaneously, this worked well and made it easier for each participant to interact when seeing not only the speaker or the shared screen, but all the faces simultaneously. All participants were asked to mute their microphone when not speaking to reduce noise, but no specific instructions regarding camera use was given. A couple of participants turned off their video-camera for some time during the session.

The active program elements of the workshop were reduced from 2 hours and 30 minutes for in-house event to 1 hour and 45 minutes for the remote program, based on the fact that digital meetings are often shorter than in-house meetings and participants speak less freely. However, we experienced to slightly running over the time of the first remote program block. Another time constraint occurred due to higher than expected degree of active participation from the users in the user feedback session. Despite the delays, a full 15 minutes break was made in between the blocks, to allow participants to completely leave the screen and take some fresh air. At the summary of the workshop it was expressed that this was appreciated and of importance for the participants. 
The organisation and preparation of the workshop were made in digital meetings for the research team in home offices. First meeting was one month before the workshop, to prepare the invitations and the program, that was sent by post mail to the participants together with a printed version of the consent form to be filled in. Later meetings targeted the program content in details. The last meeting was organised the day before the workshop, for last minutes amendments. This was experienced as an efficient way for the preparations, with meetings lasting 30-60 minutes.

\section{Discussion and Conclusion}

The main contribution of this paper lies on how to remotely apply user-centred design methodology with active contribution of end-users. The research questions (RQs) are answered based on the results.

RQ1 addressed how to perform remote user-centred design. Traditionally, in-house workshops are used for co-creation purposes and user meetings. Such arrangements can successfully be carried out with a remote digital approach, but a crucial precondition is stable internet-connectivity at home for the participants. The platform has to be carefully chosen regarding user-friendliness and the features of the screen view during the event. A collective or individual session with each participant to test-run the video conference platform, familiarise with the functionality as well as to resolve technical issues should be considered. We recommend all participants, both end-users and researchers, are instructed to mute their microphone when not speaking, but keeping the camera transmitting during the entire session as it might impact negatively on the discussion not knowing if the person is joining actively or doing other things. When entering the videoconference solution, each participant could write their name, to be visualised in the screen view. This is recommended to do for all workshop participants, also the research team, to ease the following of the discussion as all faces might not be familiar to all participants and it is easier to lead the discussion in a structured way by using the first names. Recording is recommended to ease the retrospective analysis, however attention must be paid to the storage of recordings with reference to the European privacy regulations [8]. When organising meetings with chronic pain patients, attention has to be paid on the health and safety of the participants. Physical breaks in the program schedule is such a consideration, also of importance for digital meetings. We recommend at least 10 minutes break per hour, allowing the participants to stretch the legs or leaving meeting for a while.

RQ2 asked about benefits and constraints of a digital approach. An apparent benefit of remote workshops is the participation from home, with no applied travelling time or costs. With this reduced time consumption for all parts, this approach might be used to organise user meetings with a more frequent schedule. In terms of iterative development and evaluation, the remote approach might facilitate increased user-involvement in the collection of user requirements and later on in assessment of user interface design, interactions and usability. Moreover, taking into consideration the Covid-19 pandemic, the remote approach contributes to social distancing and thereby less risk of contact spread, particularly relevant when working with users in elevated risk demographic. Regarding constraints, digital meetings offer limited social interaction between the participants, particularly if the group is unfamiliar with each other. People might speak less freely, and for that reason, the moderator(s) must actively lead the discussion by using the first names to facilitate the active voice of each participant. The recommended 
number of end-users is 6-8 persons to endeavour contribution from everyone. Digital events tend to be shorter than in-house meetings. Nevertheless, it is important to allocate enough time particularly for active user sessions. We reduced the active program schedule of the digital meeting compared to an in-house event, with the expected constraints in mind, but experienced to run out of time due to more than expected active participation from the users. Next digital workshop will be extended with another block of time. It is likely that the relative ease to get participants to be active in the workshop can partially be attributed to the fact that they had previously met in physical meetings and were familiar with the research team and the other user group members.

This study has some limitations, such as including one single workshop with a limited number of informants. However, the participants meaningfully represented the end-user group and contributed actively with their experiences. In addition, the research team has expertise in the user-centred design domain, and this paper is intended for sharing knowledge and reflections on remote procedures. This remote user-centred design methodology can be recommended for other digital health projects, and particularly usable for patients with rare diseases, as there might be large geographic distances between the participants.

Future work of the project is associated with continuation of the remote approach for co-creation user workshops and the preparation and execution of remote user evaluations, with the individual participant performing the test at home with guidance from the research team located in the control room of a usability laboratory.

\section{References}

[1] Ergonomics of human system interaction. ISO 9241-210: 2019. Part 210: Standard for human-centred design for interactive systems. International Standardization Organization (ISO), Switzerland,

[2] Gulliksen J, Göransson B, Boivie I, Blomkvist S, Persson J, Cajander Å. Key principles for user-centred systems design. Behav Inf Technol. 2003; 22:6, 397-409.

[3] Smaradottir BF. The steps of user-centered design in health information technology development. In: Proceedings of International Conference on Computational Science and Computational Intelligence; 2016 Dec, Las Vegas (NV): IEEE; p. 116-121.

[4] Bastien JC. Usability testing: a review of some methodological and technical aspects of the method. Int J Med Inform. 2010; 79(4), e18-e23.

[5] Chronic Pain: decision support for personalized chronic pain care. [cited 2020 July 1]. Available from:https://ehealthresearch.no/en/projects/decision-support-for-personalized-chronic-pain-care

[6] Norwegian Fibromyalgia Association. [cited 2020 July 1]. Available from: https://fibromyalgi.no/

[7] Smaradottir BF, Fagerlund AJ, Bellika JG. User-centred design of a mobile application for chronic pain management. Stud Health Technol Inform. 2020; 272:272-275.

[8] General Data Protection Regulation. [cited 2020 July 1]. Available from: https://ec.europa.eu/info/law/lawtopic/data-protection/data-protection-eu_en 